Asymmetric lateral coherence of betatron radiation emitted in laser-driven light sources

This content has been downloaded from IOPscience. Please scroll down to see the full text. 2015 EPL 11144003

(http://iopscience.iop.org/0295-5075/111/4/44003)

View the table of contents for this issue, or go to the journal homepage for more

Download details:

IP Address: 159.149.197.10

This content was downloaded on 15/03/2016 at 10:14

Please note that terms and conditions apply. 


\title{
Asymmetric lateral coherence of betatron radiation emitted in laser-driven light sources
}

\author{
B. Paroli $^{1}$, E. Chiadroni ${ }^{2}$, M. Ferrario ${ }^{2}$, V. Petrillo ${ }^{1}$, M. A. C. Potenza ${ }^{1}$, A. R. Rossi ${ }^{1}$, \\ L. SERAFInI ${ }^{1}$ and V. ShPAKOV ${ }^{2}$ \\ 1 Dipartimento di Fisica, Universitá degli Studi di Milano and INFN Sezione di Milano \\ via G. Celoria, 16, 20133 Milano, Italy \\ 2 INFN-LNF - via E. Fermi, 00044 Frascati, Italy
}

received 10 June 2015; accepted in final form 11 August 2015

published online 3 September 2015

PACS 41.75. Jv - Laser-driven acceleration

PACS $42.25 . \mathrm{Kb}$ - Coherence

PACS 41.60.Ap - Synchrotron radiation

\begin{abstract}
We show that the radiation emitted by betatron oscillations of a high-energy electron beam undergoing wake-field acceleration is endowed with peculiar coherence properties which deliver quantitative information about the electron trajectories. Such results are achieved by means of accurate numerical simulations and a simple geometrical model gives a clear physical interpretation.
\end{abstract}

Copyright (C) EPLA, 2015

Introduction. - Radiation sources from relativistic beams are in continuous development due to the increasing demand of shorter wavelengths and higher brightness. Soft and hard X-rays are produced at large-scale facilities as synchrotrons and linacs (by means of wigglers, bending magnets, undulators) and in compact devices as laserdriven light sources. In many experiments, the emission has been optimized, and its properties measured and characterized in view of a wide range of applications [1]. In other cases, the information encoded in the radiation has been used to understand the beam dynamics [2] or to deduce the source parameters [3-5]. Useful information can be obtained from the coherence properties. Temporal coherence depends on the strength parameter $a_{\beta}=\gamma r_{0} k_{b}$, where $\gamma$ is the Lorentz factor, $r_{0}$ is the oscillation amplitude and $k_{b}$ is the oscillation wave number. X-ray produced by sources with $a_{\beta} \gg 1$, such as magnetic wigglers or laser wake-field accelerators, are characterized by limited coherence length. Under these conditions, the analysis of the transverse spatial coherence is generally difficult or useless and the broad spectrum must be limited to get information about the bunches [6].

In this work we show that the transverse coherence of a broad spectrum radiation can nevertheless be used to characterize the dynamics of the electron beam when the radiation is emitted in a narrow cone by particles moving along trajectories with non-zero curvature, as, for example, in the case of ultrarelativistic beam sources.

The coherence of a broadband radiation can be properly defined starting from the more general definition of mutual coherence that includes information on both transverse and longitudinal coherence. This is what occurs in a general form of Young's interferometer. The cross-correlation $\Gamma_{12}(\tau)=\left\langle E\left(P_{1}, t+\tau\right) E^{*}\left(P_{2}, t\right)\right\rangle$ of the radiation diffracted by the pinholes $P_{1}$ and $P_{2}$ has an important role in determining the visibility of the observed fringes and both temporal and spatial coherence effects play a role (here $\tau$ is a time delay). The envelope of the fringe pattern at zero pathlength differences is an indication of spatial coherence effects, while the decreasing of visibility at different pathlength is an effect of the temporal coherence. For a good evaluation of spatial coherence it is necessary to make temporal coherence effects negligible (quasi-monochromatic conditions). In this case the mutual coherence function $\Gamma_{12}(\tau)$ reduces to the mutual intensity $J_{12} e^{-j 2 \pi \bar{\nu} \tau}$, where $J_{12}=\Gamma_{12}(0)$ and $\bar{\nu}$ is the central frequency of the quasimonochromatic radiation. For broadband radiation the normalized mutual coherence function, by assuming $\tau=0$ elsewhere, is characterized by peculiar properties. In fact, the transverse coherence measured around a proper offaxis fixed point is proven here to have a characteristic asymmetric enlargement with two additional peaks with respect to that measured around the average motion axis. 


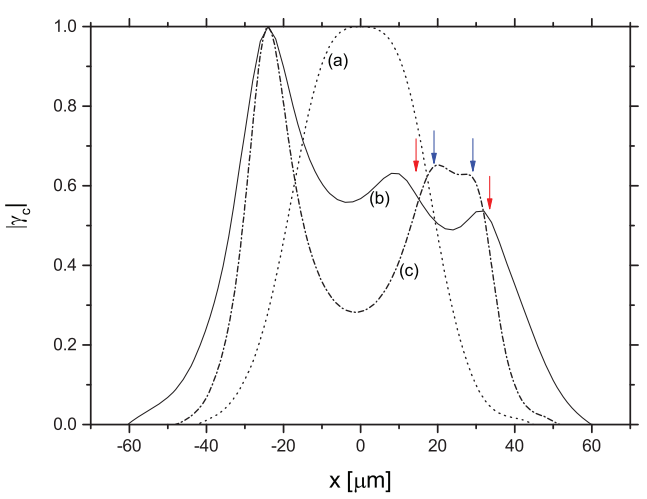

Fig. 1: (Colour on-line) Simulations of the radiation coherence factor $\left|\gamma_{c}\right|$ with $\tau=0, \vec{X}_{0}=(-24 \mu \mathrm{m}, 0,1 \mathrm{~m}), \vec{X}=$ $(x, 0,1 \mathrm{~m})$ for electrons oscillating in betatron motion with average amplitude $>5 \mu \mathrm{m}$ (solid line) and $<3 \mu \mathrm{m}$ (dashed-dotted line). The virtual detector has $64 \times 64$ cells with a 16 bit time resolution. In order to reduce the computational time, the coherence was calculated for a longitudinal slice extended over $1 / 20$ of the entire beam $\left(10^{3}\right.$ particles of $\left.2 \cdot 10^{4}\right)$. The field emitted from a particle at a distance of $1 / 20$ of the bunch length is temporally separated by a particle in the adopted slice. The dashed line is the coherent factor obtained around the longitudinal axis $\vec{X}_{0}=(0,0,1 \mathrm{~m})$ and with particles oscillating with average amplitude $>5 \mu \mathrm{m}$. The arrows are the peak positions predicted by the model with oscillation amplitude of $5 \mu \mathrm{m}$ (red) and $3 \mu \mathrm{m}$ (blue).

Such enlargement depends on elongation and curvature of the particle trajectories. As a result, a measurement of the transverse coherence gives statistical information about the dynamics of electrons undergoing oscillatory motions, with direct applications in particles beam and radiation source diagnostics. We analyze the results of numerical simulations describing a laser-driven electron beam moving in transverse betatron oscillations.

Simulation method. - Betatron motion was simulated with Q-Fluid [7], a hybrid PIC-Fluid code for simulation of plasma wake-field acceleration. A low-emittance $(2.7 \mathrm{~mm} \mathrm{mrad}) 14 \mathrm{pC}$ electron beam with a $3.5 \mu \mathrm{m}$ size and $78 \mathrm{MeV}$ initial energy, accelerated in a potential well produced in a $10^{17} \mathrm{~cm}^{-3}$ dense plasma was considered, these values being similar to the nominal parameters of the SPARC_LAB source at the National Laboratories of Frascati (LNF) [8]. The radiated field is obtained by computing the Lienard-Wiechert potentials on a virtual detector. Figure 1 shows a section, along the transverse coordinate $x$, of the modulus of the complex degree of coherence:

$$
\gamma_{c}(\tau)=\frac{\Gamma\left(\vec{X} ; \vec{X}_{0} ; \tau\right)}{\left[\Gamma\left(\vec{X}_{0} ; \vec{X}_{0} ; 0\right) \Gamma(\vec{X} ; \vec{X} ; 0)\right]^{1 / 2}},
$$

where

$$
\Gamma\left(\vec{X} ; \vec{X}_{0} ; \tau\right)=\lim _{T \rightarrow \infty} \frac{1}{2 T} \int_{-T}^{T} E(\vec{X}, t+\tau) E^{*}\left(\vec{X}_{0}, t\right) \mathrm{d} t
$$

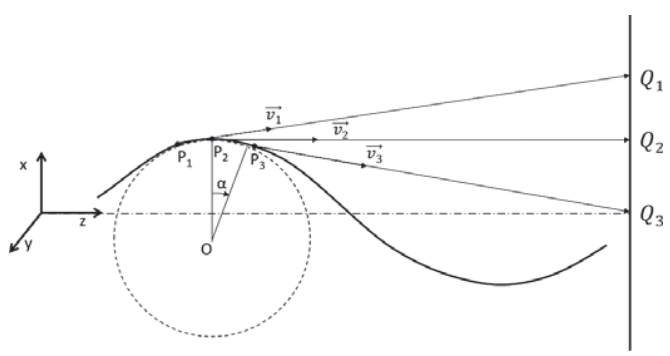

Fig. 2: Geometrical interpretation of the coherence features. The oscillating electron trajectory (solid line) is locally approximated by its osculator circle (dashed line) at the maximum transversal elongation position. The electron emission at positions $P_{i}, i=1,2,3$, with direction $\overrightarrow{v_{i}}$ is represented. The radiation is observed onto a plane screen transverse to the $z$-axis in the positions $Q_{i} . Q_{2}$ receives radiation with the shortest optical path.

is the cross-correlation function of the electric field $E$ (x-component). Here $\tau=0$, the point $\vec{X}=(x, y, z)$ is referred to a Cartesian frame with the $z$-axis along the average direction of the electron beam (fig. 2), while $\vec{X}_{0}$ is a fixed point on the detection plane. For clarity we will refer to $\gamma_{c}$ as the complex coherence factor $\gamma_{c}=\gamma_{c}(0)$ also when the radiation is not really quasi-monochromatic.

No peculiar properties of coherence related to electron trajectory can be revealed when we consider the fixed point $\vec{X}_{0}$ (see eq. (1)) corresponding to the axis $\left(x_{0} \approx 0, y_{0} \approx 0\right)$ (curve (a) of fig. 1 ). In such condition, the transverse coherence is limited by the temporal coherence, because the coherence length $\approx 1 \mathrm{~nm}$ (corresponding to the critical frequency $\omega_{c} \hbar \approx 1 \mathrm{keV}$ ) is comparable to the length of the extra path $\sqrt{z_{0}^{2}+\left(S_{c} / 2\right)^{2}}-z_{0}$ from the radiation source to the detector, $S_{c} \approx 40 \mu \mathrm{m}$ being the Full Width at Half-Maximum (FWHM) of $\left|\gamma_{c}\right|$. On the contrary, when $\vec{X}_{0}$ is far away from the longitudinal axis, the transverse coherence of the radiation shows multiple oscillations and the coherence factor has a faster decrease, depending on the average amplitude of the trajectories. In fig. 1 the coherence factor $\left|\gamma_{c}\right|$ is computed around a fixed point $\vec{X}_{0}$ at a distance of $-24 \mu \mathrm{m}$ from the longitudinal axis and at $1 \mathrm{~m}$ from the beam initial position (the distance of the fixed point from the axis must satisfy the condition that will be discussed in the model below) (see curves (b) and (c)). The presence of these peaks does not depend on the number of particles used in simulations, which ranges from $10^{3}$ up to $10^{6}$. This last case was restricted to particles with trajectories in the $x-z$ plane and with both phase and amplitude uniformly distributed in the ranges from $-30 / \gamma^{3}$ to $30 / \gamma^{3} \operatorname{rad}$ and from 5 to $6 \mu \mathrm{m}$, respectively.

Single-particle model. - In the ideal limit $\gamma \rightarrow \infty$, the radiation is instantaneously emitted along the direction of the normalized particle velocity $\vec{\beta}=\vec{v} / c$, in a cone with vanishing width $1 / \gamma$. Each point of the screen where the radiation is detected is in biunivocal correspondence 
with the point of the trajectory from which the radiation was emitted. In a more realistic, finite $\gamma$ case, the radiation illuminates a finite region on the screen identified by a cone of aperture $1 / \gamma$, whose vertex is at the emission point and whose axis is parallel to the electron velocity $\vec{v}$. A simplified geometrical interpretation of the coherence features described above can be obtained by studying a single particle in a half-cycle of a betatron oscillation.

The particle trajectory around the maximum $x$ elongation is locally approximated by its osculator circle with radius $R$. In fig. 2, the emission at three subsequent times, $t_{i}, i=1,2,3$, corresponding to the electron positions $P_{i}$, is considered. Each position is identified by an angle $\alpha_{i}$ at the center of the circle, with $\alpha_{2}=0$. The positions $Q_{i}$ are the intersections of the emission directions $\vec{\beta}_{i}$ with the observation plane. In general, each parameter can be expressed as a function of the continuous variable $\alpha$, where the condition $\alpha=0$ now identifies the osculation point introduced above. The geometrical connection between each trajectory point $P(\alpha)$ and the corresponding points on the screen $Q(\alpha)$ is given by the bijection $x_{Q}(\alpha)=\tan \alpha(R \sin \alpha-z)+R \cos \alpha$. Point $Q(\alpha)$ is then completely determined. The distance between the two points $P(\alpha)$ and $Q(\alpha)$ is

$$
L(\alpha)=\sqrt{(z-R \sin \alpha)^{2}+\left[R \cos \alpha-x_{Q}(\alpha)\right]^{2}} .
$$

The function $L(\alpha)$ has a minimum in $\alpha=0$, as can be seen by assuming $z \gg R$ or $\alpha \ll 1$. As a consequence, the corresponding observation point $Q(0)$ will be the first to receive radiation, the radiation emitted for $\alpha \neq 0$ taking a longer time to reach the screen. Coming back to the example of fig. 2, we can observe that each point $Q_{i}$, with coordinate $x_{Q i}$ on the screen, receives light with different delays from the whole trajectory and, in particular, from the three positions $P_{i}$. Furthermore, there is a position $x_{s}$ where the radiation from $P_{1}$ and $P_{3}$ will be received simultaneously, i.e. $L\left(\alpha_{3}\right) / c+t_{3}-t_{1}=L\left(\alpha_{1}\right) / c$. Assuming the velocity of the particle to be almost constant around the osculation point, the radiation intensity within each $1 / \gamma$ cone emitted by both points turns out to be approximately the same. When $\vec{X}_{0}=Q_{1}$, a second peak of the coherence factor is then expected at the point $Q_{3}$. The radiation observed simultaneously at points $Q_{1}$ and $Q_{3}$ is the origin of the asymmetric enlargement observed in simulations. In particular, the second maximum is given by the radiation emitted from oscillation in the half-plane $x-z$ containing the fixed point, while the third maximum is given by the radiation from oscillation in the opposite half-plane $x-z$. Radiation at each peak position is simultaneously observed at the fixed point. The arrows in fig. 1 show the peak positions foreseen by the model.

The fast decreasing of the coherence factor close to the fixed point is due to the asymmetric detection method. In fact, when the fixed point $\vec{X}_{0}$ is close to $Q(0)$, corresponding to the minimum of $L(\alpha)$, the derivative $\mathrm{d} L(\alpha) / \mathrm{d} \alpha$ is close to zero and the variation $\Delta L$ of the optical path $L(\alpha)$ from $Q(0)$ to the point $\left(x_{s}, y_{0}, z_{0}\right)$ is lower than the coherence length $L_{c}$ of the radiation, so that the coherence factor $\left|\gamma_{c}\right|$ does not change appreciably. On the contrary, when $\vec{X}_{0}$ is far enough from $Q(0)$, the derivative $\mathrm{d} L(\alpha) / \mathrm{d} \alpha$ increases appreciably and $\Delta L$ is larger than $L_{c}$. When the fixed point is such that the condition $\Delta L>L_{c}$ is satisfied, $\left|\gamma_{c}\right|$ changes rapidly, bringing information about the elongation of the electron trajectories. In particular, the decrease of $\left|\gamma_{c}\right|$ is determined by both the fixed point and the transverse amplitude of the oscillations. The results of simulations in fig. 1 show that $\left|\gamma_{c}\right|$ decreases faster for electrons oscillating with average amplitude $\overline{r_{p}}<3 \mu \mathrm{m}$ with respect to those with $\overline{r_{p}}>5 \mu \mathrm{m}$. Here $\overline{r_{p}}=\frac{1}{T} \int \sqrt{x_{p}(t)^{2}+y_{p}(t)^{2}} \mathrm{~d} t$, where $x_{p}$ and $y_{p}$ are the transverse coordinates of particles. The model predicts that around $x_{0}=-24 \mu \mathrm{m}$ the derivative $\mathrm{d} L(\alpha) / \mathrm{d} \alpha$ for electrons oscillating with $\overline{r_{p}}<3 \mu \mathrm{m}$ is larger than that for $\overline{r_{p}}>5 \mu \mathrm{m}$. The decoherence is then faster in the former case with respect to the latter, in agreement with our simulations.

Even if the positions of the two peaks in fig. 1 are in good agreement with those predicted by the model, different limitations of the single-particle model must be taken into account. The first limit is given by the transverse spatial distribution of an ensemble of particles that can substantially change the shape of $\left|\gamma_{c}\right|$. In particular, when the radiation is mainly emitted by particles moving close to the acceleration axis, the two peaks cannot be easily resolved. According to our model this is what occurs for particles with oscillation amplitude lower than $2 \mu \mathrm{m}$. On the contrary, for larger amplitudes of oscillation, betatron emission mechanism promotes the resolution of the two peaks. This occurs because particles oscillating close to the axis emit radiation with lower intensity with respect to particles at the edge, due to the higher transverse acceleration of the latter with respect to the former. The longitudinal distribution of particles gives a stringent limitation on the shape of $\left|\gamma_{c}\right|$ when the assumption of ergodicity of eq. (1) is not fully satisfied. A priori non-stationary analysis of the process should be taken into account as in ref. [9], however under the assumption of ergodicity the statistical properties of the process are simplified. We assume that the beam properties in the simulated slice has the same statistical properties of the other slices and we verified that the ensemble of particles used in simulation is temporally uncoupled with the adjacent slices, i.e. the condition $\Delta t \gg \tau_{c}$ holds, where $\Delta t$ is the time duration of the radiation pulse and $\tau_{c}$ is the characteristic coherence time of radiation. Another limitation of the single-particle model is that it should take into account the energy spread of particles. We have estimated that a variation of $20 \%$ in the particle momentum change the width (FWHM) of peaks up to $80 \%$.

Quasi-monochromatic radiation. - When the radiation is considered quasi-monochromatic, the limit 


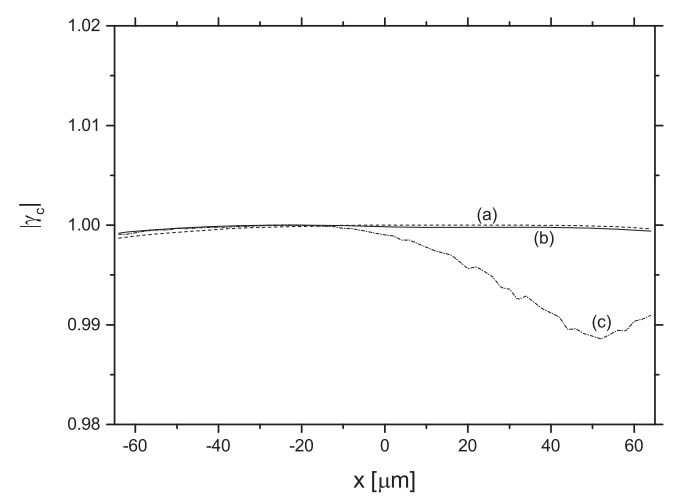

Fig. 3: Modulus of $\gamma_{c}$ for a quasi-monochromatic radiation with $\vec{X}_{0}=(-24 \mu \mathrm{m}, 0,1 \mathrm{~m})$ for particles oscillating with average amplitude $>5 \mu \mathrm{m}$ (solid line (b)) and $<3 \mu \mathrm{m}$ (dashed-dotted line (c)). Dashed line (a) is the modulus of $\gamma_{c}$ with $\vec{X}_{0}=$ $(0,0,1 \mathrm{~m})$ and with particles oscillating with average amplitude $>5 \mu \mathrm{m}$.

$\Delta_{L}>L_{c}$ discussed above is not satisfied and the modulus of $\gamma_{c}$ must show a plateau $\approx 1$ for each value of $\vec{X}$, until $\vec{X}$ is close to $\vec{X}_{0}$. It will decrease (in far field) with a characteristic length $\left|x-x_{0}\right| \approx \bar{\lambda} z_{0} / 2 r_{p}$ in accordance with the characteristic size of the transverse coherence for monochromatic radiation [10]. The same behavior occurs with a broad spectrum when again the limit $\Delta_{L}>L_{c}$ is not fully satisfied, for example because the fixed point $\vec{X}_{0}$ is on-axis. In fact the coherence factor decreases monotonically when the temporal coherence length of radiation $L_{c}$ approaches the extra path $\sqrt{z_{0}^{2}+x^{2}}-z_{0}$ (this is the case of curve (a) in fig. 1). We give a quantitative estimate of such considerations showing the same results of fig. 1 for a quasi-monochromatic radiation with average wavelength $\bar{\lambda}=4 \mathrm{~nm}$ (see fig. 3 ). The radiation is obtained filtering with a narrow band-pass filter the simulated betatron radiation. The modulus of $\gamma_{c}$ has a constant value close to unity, i.e. a high degree of coherence in the simulated spatial range. This is in agreement with the expected transverse coherence length $\approx 500 \mu \mathrm{m}$ that is greater than the observable range of simulation (from $x=-64 \mu \mathrm{m}$ to $x=64 \mu \mathrm{m})$. The variation of the curve (c) of about $1 \%$ is not significant due to the limited number of particles used in simulation $\left(10^{3}\right.$ particles $)$. We conclude that the broad spectrum of emission is fundamental to the formation of the two additional peaks on the modulus of $\gamma_{c}$.

Conclusions. - We have shown that the coherence factor has two additional maxima when it is characterized with respect to a fixed point. The fixed point must be such that the condition $\Delta L>L_{c}$ is verified for each particle. The coherence factor is characterized by peculiar properties which are not present around the motion axis. Such properties are well described by a single-particle geometrical model. This method gives information about the wavefront of the radiation emitted by particles oscillating at least in a half-cycle. Such information is related to various observable quantities: i) the variation of the coherence factor around the fixed point (main peak) that directly depends on the derivative of the optical path $\mathrm{d} L(\alpha) / \mathrm{d} \alpha$ imposed by each particle trajectory. The measurement of the coherence factor size around the fixed point is thus correlated in statistical sense with the transverse amplitude of electron oscillations. ii) Multiple oscillations of the coherence factor related to the oscillatory behavior of particles. This information is of remarkable interest to characterize the beam dynamics.

The coherence behavior discussed in this work can give insight in the beam properties for a wide range of sources. It will be applied to the laser-driven plasma acceleration experiment under development at SPARC_LAB, LNF. In particular the width of the electron beam affects the shape and position of the additional maxima. When at large distance $L(\alpha)$ becomes independent of $R$ the second and third peak positions depend only on the transverse beam size. As predicted by the model the positions of the first and second maxima are roughly expected at $-\operatorname{sign}\left(x_{0}\right)\left|2 r_{p}-x_{0}\right|$ and $-\operatorname{sign}\left(x_{0}\right)\left|2 r_{p}+x_{0}\right|$, respectively (see blue and red arrows in fig. 1 ). Here the $x$-component of the fixed point is $\left|x_{0}\right|>2 r_{p}$. The shape of the coherence factor also changes because the two peaks are more spread out when the beam size increases, as shown in fig. 1. On the contrary, the peak positions of the coherence factor are not very sensitive to the momentum spread of the beam. Our estimation comes from the single-particle case in which a reduction of the particle momentum (with $\gamma=1000)$ of $\approx 20 \%$ introduces variations less than $2 \%$ in the peak positions while it gives substantial variations of $\approx 80 \%$ in the peak widths (FWHM).

Other possible applications are in ultrarelativistic beam sources like in third generation synchrotrons, where the curvature of particles and the broad spectrum of radiation are imposed by means of bending magnets or by insertion devices like wigglers. We plan to exploit this method for particle beam diagnostics in collaboration with the Beam Instrumentation Group at CERN, with the aim of operating on ultrarelativistic electron beams and in the LHC proton beam.

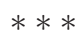

This work was supported by the Italian Ministry for University and Research (MIUR) "FIRB 2012" funds (grant No. RBFR12NK5K).

\section{REFERENCES}

[1] Malka V. et al., Nat. Phys., 4 (2008) 447.

[2] Phuoc K. T. et al., Phys. Rev. Lett., 104 (2010) 225501.

[3] Alaimo M. D. et al., Phys. Rev. Lett., 103 (2009) 194805.

[4] Alaimo M. D. et al., Opt. Express, 22 (2014) 30013. 
[5] Yabashi M., Tamasaku K. and Ishikawa T., Phys. Rev. Lett., 87 (2001) 140801.

[6] Kohn V., Snigireva I. and Snigirev A., Phys. Rev. Lett., 85 (2000) 2745.

[7] Rossi A. et al., Nucl. Instrum. Methods Phys. Res. A, 740 (2014) 60.
[8] Ferrario M., Alesini D., Anania M. et al., Nucl. Instrum. Methods Phys. Res. B, 309 (2008) 183.

[9] Geloni G. et al., Understanding transverse coherence properties of X-ray beams in third generation SR source, DESY 05-109, ISSN 0418-9833 (2005).

[10] KneIP S. et al., Nat. Phys., 6 (2010) 980. 